\title{
Two Cases of Dog Bite with Similar Clinical Presentation but Outcome Variation - A Case Report
}

\author{
MD ROBED AMIN, ${ }^{1}$ SHAIKAT CHOWDHURY ${ }^{2}$ SHABNAMFATEMA ENAM, ${ }^{3}$ SAFINARAFATH RAHMAN, ${ }^{4}$ AHMED \\ RIYADH HOSSAIN, ${ }^{5}$ M SALIMUZZAMAN, ${ }^{6}$ MD AZIZUL KAHHAR ${ }^{7}$
}

\section{Introduction:}

Paralytic rabies was initially recorded in $1887^{1}$ but not widely identified until decades later ${ }^{2,3}$. Paralytic rabies continues to be confused with Guillain-Barr'e syndrome (GBS) and related disorders, treatable autoimmune diseases of peripheral nerves. Misdiagnoses of rabies have led to humanto-human transmission through corneal, liver, and kidney transplants from donors who were thought to have GBS or stroke ${ }^{4}$. Many rabies patients misdiagnosed as GBS have undergone plasma exchange ${ }^{5}$. Clinically the classical furious form having hydrophobia, aerophobia, laryngospasm and autonomic disturbance are not common in paralytic rabies Survival time in paralytic rabies is longer than in furious rabies $^{6}$. In vaccine preventable era, the highly efficacious cell culture vaccine is still need assistance with human rabies immunoglobulin for complete protection especially in CAT III wound injury ${ }^{7}$. Although WHO declared to phase of the NTV(Nerve Tissue Vaccine) due to its inefficiency and chance of serious adverse event, the developing country like Bangladesh and Pakistan is still using the drug with an aim to phase it out slowly ${ }^{8}$. The NRV induced encephalomyelitis is well documented in literatures and case reports and clinical features of which may confuse with paralytic rabies as well. Here is two cases of dog bite presented in Dhaka Medical College Hospital(DMCH) with similar clinical presentation but later diagnosed as separate disease and different outcome.

\section{Case 1:}

Mr. AA, a 50 years old farmer from Gazipur was admitted in Medicine unit, Dhaka Medical College Hospital with the complaints of fever for 15 days, weakness of the both lower limbs for 10 days, restlessness, irrelevant talk followed by

1. Associate Professor of Medicine, Dhaka Medical College

2. Medical Officer, Dhaka Medical College

3. Honorary Medical Officer, Dhaka Medical College

4. Honorary Medical officer, Dhaka Medical College

5. Assistant Registrar, Dhaka Medical College

6. Infectious Disease and Tropical Medicine Specialist, OSD, DGHS

7. Professor of Medicine, Dhaka Mecial College

Correspondence: Dr.Md Robed Amin, Associate Professor of Medicine, Dhaka Medical College, Apt-C-2, House-76, Block-F, Road-5, Bonani, Dhaka. Email-robedamin@yahoo.com drowsiness for 5 days.Fever was high grade, intermittent at onset, later continuous in nature. Five days after the onset of fever the developed lower limb weakness. Weakness gradually evolved into complete paralysis of both lower limbs. He also developed acute retention of urine within this period. He has no $\mathrm{H} / \mathrm{O}$ headache, vomiting convulsion, hydrophobia, etc.

According to the patient's attendant, he was reasonably well 1 month back. While working in the corp field he was bitten by a stray dog. There were multiple transdermal bites over the lateral aspect of the left ankle joint and multiple scratches over the left palm. The bite was unprovoked and of category III. The stray dog bitten several persons in the vicinity and later it was beaten to death by concerned people in the village.

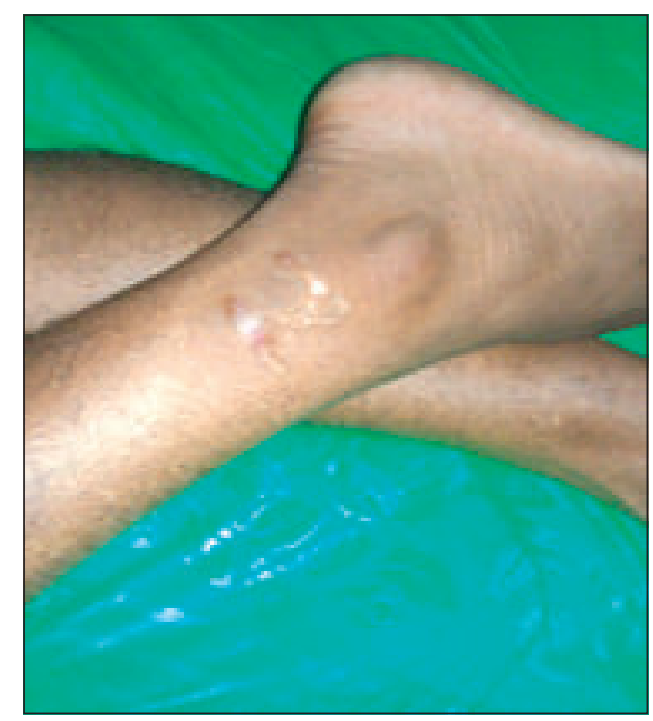

Fig 1: The bite mark of CATIII type injury

He applied some herbs over the bite wound to stop bleeding and rushed to the nearest hospital. There attending physician advised him for wound toileting with soap water and cell culture vaccine (Inj. Verorab) IM according to Essen schedule $(0,3,7,14,28)$. He took Inj. Verorab on that day and thoroughly washed his wound with soap. After taking $4^{\text {th }}$ dose of Inj. Verorab(on day 14)he began to develop fever followed by weakness of both lower limbs and clouding of consciousness gradually. He was treated with Inj Antibiotics 
and High dose steroids in infectious disease hospital . As there was no improvement observed,he was referred to the medicine unit, DMCH.

On general examination he was toxic, not anaemic, nonicteric, body temerature of $101^{\circ} \mathrm{F}$, pulse $100 / \mathrm{min}, \mathrm{BP}-110 / 80 \mathrm{mHg}$, His GCS score was $7\left(\mathrm{E}_{1} \mathrm{~V}_{2} \mathrm{M}_{4}\right)$, no sign of meningism, cranial nerves couldn't be evaluated, all jerks absent, plantar bilateral equivocal, muscle power couldn't be evaluated. Respiratory system examination revealed bilateral coarse crackles. Other system examination revealed nothing significant.

Investigation showed - Hb-10.8 g/dl, ESR33 mm, Platelet 150000/L, Leukocyte count 6900/L, Neutrophil 90\%, Lymphocyte 6\%, SGPT 48 u/l, S. Creatinine $1.1 \mathrm{mg} . \mathrm{dl}, \mathrm{S}$. $\mathrm{Na}^{+} 136 \mathrm{mmol} / 1, \mathrm{~S} . \mathrm{K}^{+} 4.4 \mathrm{mmol} / \mathrm{l}, \mathrm{CSF}$ study: Protein 131 $\mathrm{mg} / \mathrm{dl}$, Sugar $116 \mathrm{mg} / \mathrm{dl}$, WBC 4/mm $\mathrm{mm}^{3}$, Lymphocytes $90 \%$,Neutrophils $10 \%$, on Gram's stain and ZN stain no organism seen and VDRL negative. NCV study showeddemylination and remylination and axonal injury in peripheral nerves.PCR of csf could not be done due to lack of facility.MRI revealed Hyperdensity in subcortical territory and brain stem. Serum antibody test(IFA) for rabies, REEFIT test in CSF and virus neutralizing antibody(VNA) could not be done due to lack of facility. Viral RNA detection through neck skin and saliva was attempted but could not be done due to his progressive deteriorating clinical course.

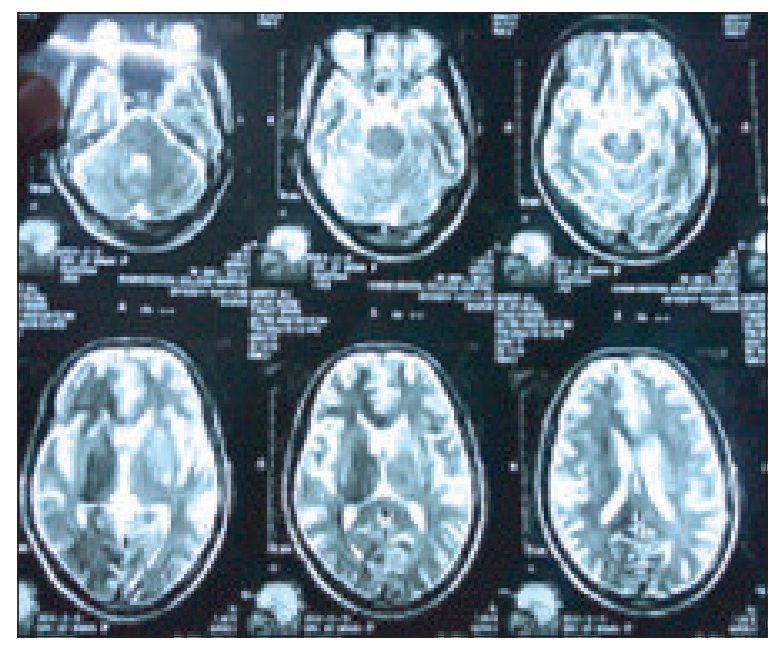

Fig.-2: MRI revealed Hyperdensity in subcortical territory and brain stem

He was given symptomatic treatment with Inj. Ceftriaxone, Inj. Metronidazole and other medication. There was no improvement of his consciousness rather he deteriorated gradually and GCS become 3 after 72 hours. The patient developed decerebrate posturing followed by central pontine hyperventilation. He was diagnosed as a case of Paralytic Rabies with Respiratory paralysis. Consultation with rabies expertise was seek home and abroad. There was constant hypersecretion of saliva throughout the course of his illness. There was refusal of admission the case in ICU facility due to unavailability of bed. There was no improvement in respiratory failure through the conservative management by respiratory stimulant. There was no hydrophobia or aerophobia during course of illness but penile tumescence was observed during hospital stay. Milwaukee protocol was not tried in this case as the case was paralytic rabies rather furious one. Seven days after admission into $\mathrm{DMCH}$ he succumbed to death due to respiratory failure. Autopsy was not done due to lack of agreement by attendant.

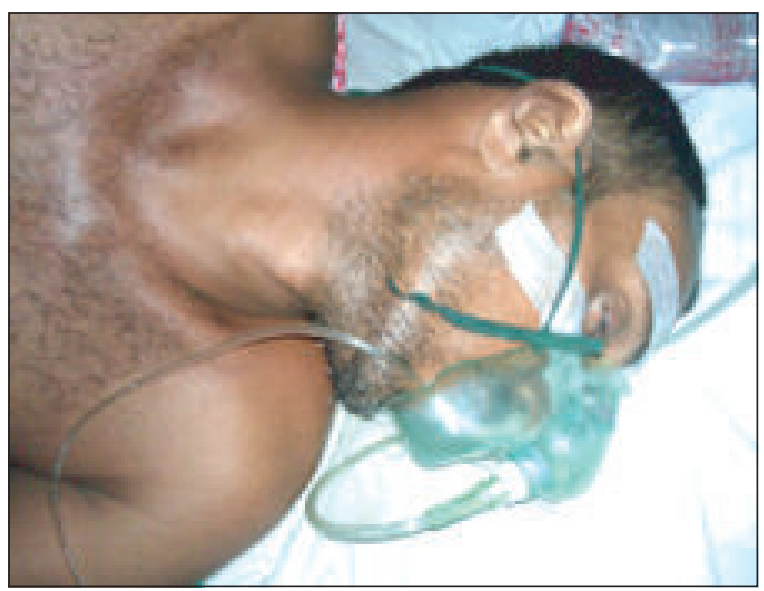

Fig.-3: Pt developed paralytic rabies with respiratory failure

\section{Case 2:}

Mr. S, a 14 years old student from Manikgonj was admitted in Medicine unit, Dhaka Medical College Hospital with the complaints of high grade, continued fever for 5 days, weakness of all the four limbs for 4 days, irrelevant talk followed by drowsiness for 2 days.

According to the patient's attendant he was reasonably well 1 month back. Then he was bitten by a stray dog over the dorsum of the rt-foot while walking along the road. The bite was single transdermal, provokedand of category III. Thedog couldn't be traced after the event. Immediately he rushed to the nearby hospital where his wound was washed with antiseptic solution (Savlon). The wound was left unsutured and no anti rabies vaccine or rabies immunoglobulin was advised. He didn't apply any type of local irritants like herbs upon the wound. 


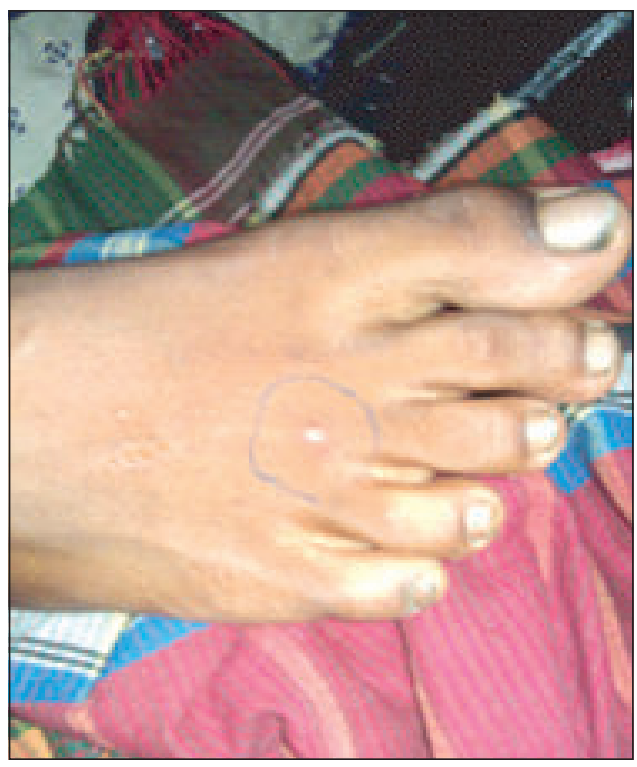

Fig.-4: The bite mark of the $2^{\text {nd }}$ case (Cat III injury)

1 week after dog bite he went to the Infectious disease hospital, Mohakhali, Dhaka and collected 14 doses of nervous tissue vaccine altogether.He began to take each dose of vaccine around the umbilicus each day by a local pharmacist. After taking 10 doses, vaccine he developed fever - high grade, persisting for 1 day and then subsided. He stopped to take vaccine for the next 2 days. After 2 days, he again began to take vaccine. After taking 12 doses of vaccine, he developed fever, weakness of all the four limbs and clouding of consciousness. He has no H/O headache, convulsion, vomiting, hydrophobia, etc.

With these complaints he was admitted into infectious disease hospital, Dhaka. There physical examination revealed temp $101^{0} \mathrm{~F}$, neck stiffness $(+)$, Kernig's sign $(+)$. There, he had been diagnosed clinically as a case of bacterial meningitis though CSF study not done and treated with Inj. Ceftriaxone and Inj. Oradexone for 3 days. His physical condition not being improved he was referred to the medicine unit, $\mathrm{DMCH}$.

In $\mathrm{DMCH}, \mathrm{General}$ examination revealed temperature normal, pulse $-70 / \mathrm{min}$, BP-90/60 mmHg. Nervous examination revealed GCS- $9\left(\mathrm{E}_{2} \mathrm{~V}_{4} \mathrm{M}_{3}\right)$, Pupil dilated, reacting to light, cranial nerves can't be evaluated, muscle tone hypotonicity in all four limbs, all jerks diminished, plantar - equivocal on both side. Other systemic examination reveals nothing significant. A presumptive diagnosis was thought about brain stem encephalitis due to paralytic rabies or acute disseminated encephalomyelitis due to nerve tissue vaccine.

Investigation showed S. creatinine- $0.74 \mathrm{mg} / \mathrm{dl}, \mathrm{S} . \mathrm{Na}^{+}-144$ $\mathrm{mmol} / \mathrm{L}, \mathrm{K}^{+}-5.1 \mathrm{mmol} / \mathrm{L}, \mathrm{CSF}$ study: appearance - hazy, total count of WBC $-20 / \mathrm{mm}^{3}$, lymphocyte- $100 \%$, neutrophil $-0 \%$, sugar $-97 \mathrm{mg} / \mathrm{dl}$, protein $-65 \mathrm{mg} / \mathrm{dl}$, Gram stain and ZN stain - no organism seen, CSF culture yielded growth of Actinobacter species (moderate growth), VDRLnegative.MRI of the brain - Features Suggestive of Acute Dimyelination in cerebral cortex and brain stem.

He was diagnosed as a case of Acute Disseminated Encephalitis due to nerve tissue vaccine.He was treated with Inj. Ceftriaxone, Inj. Methyl Prednisolone followed by oral prednisolone. He recovered from unconsciousness within $24 \mathrm{hrs}$ of Methylprednisolone but developed Ptosis of left eye. His muscle power and tone returns normal within two weeks of getting high dose steroids. Pt was able to walk with on foot at second week of hospital stay. His ptosis disappeared completely and pt was treated with cell culture vaccine according to zagreb schedule $(0,7,14)$. He improved clinically later and was discharged from the hospital.

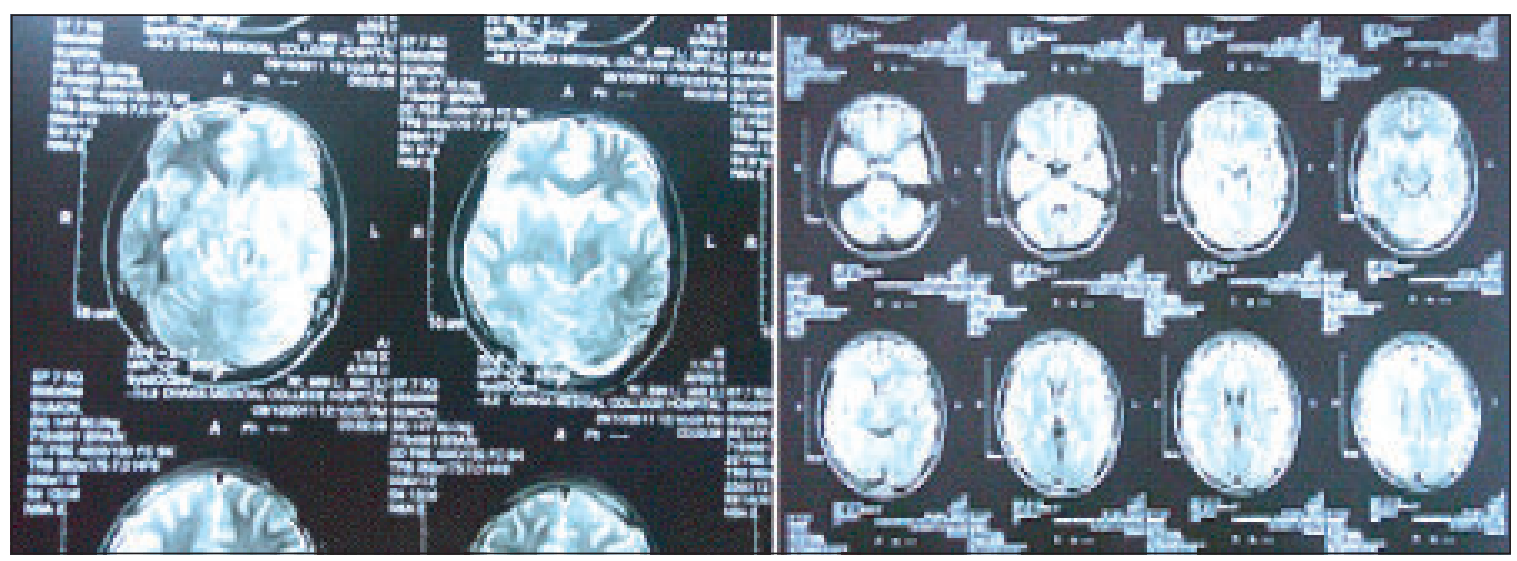

Fig.-5: MRI revealed dimyelination in brain stem and cortex of both hemisphere. 


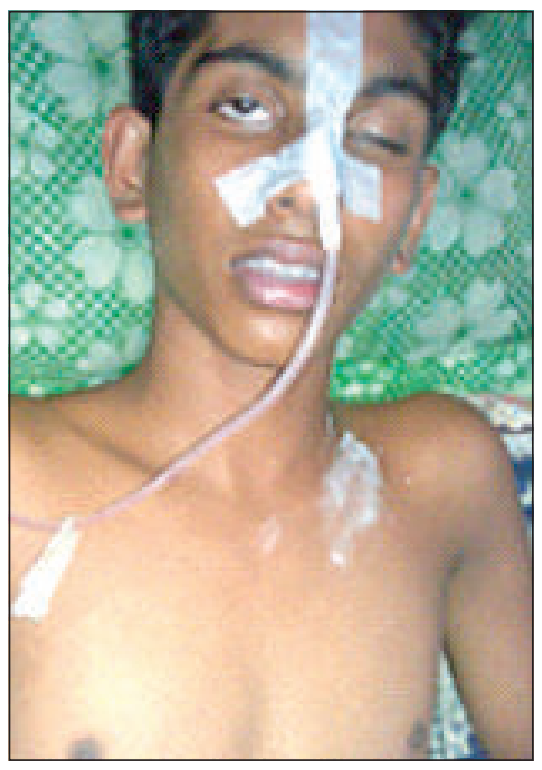

Fig.-6: Patient developed ptosis and then with treatment completely recovered.

\section{Discussion:}

Furious rabies is a well-recognized clinical disorder in humans but the paralytic form is not as easily identified.Paralytic rabies continues to be confused with Guillain-Barr'e syndrome(GBS) and related disorders ${ }^{9,10}$. Cardinal features of furious rabies, fluctuating consciousness, hydro- or aerophobia andinspiratory spasms, signs of autonomic dysfunction are not commonly seen in paralytic rabies patients $^{10}$

Only one or two classical signs of rabies, or even none, may be seen during the whole clinical course in paralytic rabies. Consciousness is usually preserved until the preterminal phase. Phobic spasms are reported in only half of confirmed paralytic rabies patients ${ }^{11}$. Weakness is the initial manifestation in paralytic rabies, whereas this was noted only when furious rabies patient approached coma. Classically the paralytic rabies patient presented with Fever, local prodrome in $1 / 3$ cases, phobic spasms in $1 / 2$ cases, Rare distal paresthesias, percussion myoedema Ascending weakness, may start at bitten limb. Average survival period 11 days (versus 6 days in furious rabies) ${ }^{10}$.In our first cases the characteristics fever and distal parasthesia was the initiating symptoms followed by weakness. Only uncommon presentation was his consciousness which also started to decline quickly and the longevity was $9^{\text {th }}$ day after the symptoms. Absence of preceding infection, absence of ascending paralysis followed by proximal weakness, absence of cranial neuropathy and early unconsciousness makes GBS unlikely possibility and the rapid worse scenario make the diagnosis likely to be paralytic rabies.
MRI findings of the brain were similar in five rabies patients (two furious and three paralytic) and demonstrated as illdefined, mild hyper intensity changes in the brain stem, hippocampi, hypothalami, deep and subcortical white matter, and deep and cortical gray matter

on T2-weighted images ${ }^{12}$. In our case there was also hyper intensity noted in subcortical region and also at brain stem .Multifocal demyelination along with length-dependent motor or sensory neuropathy is a common finding in NCV study which was also seen in our paralytic rabies patient but the $\mathrm{F}$ wave latency consistent finding of GBS was absent. The peculiarity of the paralytic case in this states that there was no protection found in receiving four IM doses of CCV which indicates the importance of HRIG in every case of dog bite having CAT II injury.

The second case was classical presentation of vaccine induce Encephalomeylitis which is reported in different literatures ${ }^{13,14}$. The presentation was also very similar like the paralytic rabies case, but it is more common with NTV rather $\mathrm{CCV}$ which the second case received.NTV contain myelin, which is responsible for the neuroparalytic side effects of these vaccines. The incidence of neural complications with vaccines prepared from brains ofadult animals is between 1:200. These complications usually occur after the sixth or seventhinjection of the vaccine. The onset of symptoms is usually within 30 days ( $88 \%$ within 20 days $)^{14}$. The condition mayresult in serious residual paralysis and even death. Thereis no definite treatment of these neuroparalyticcomplications, but high dose corticosteroids, plasma exchange and immunoglobulins have been used withvariable results. In our second case the scenario is consistent although his manifestation developed after getting 12 days of semple vaccine. Although there was serious neuroparalysis during presentation, the response with high dose steroids was excellent in case vignette rather variable findings in literature. The patient able to initiate walking with aids in two weeks was observed and complete recovery was seen within two months. The response to methylprednisolone indicates the disease as having immune mediated injury and not to be a case of GBS. The MRI findings were consistent with ADEM. Although literature revealed different types of presentation of vaccine induced neuroparalysis likeMeningoencephalitis, Meningoencephelomyelitis, Mononeuritis multiplex, Dorso-lumbar transverse myelitis and Ascending paralysis of Landry's type rabies ${ }^{15}$, in our case it was found to have encephalomyelitis variety. The CSF was having mild increase in protein while cell count was only 20 indicating no infective agent having meninges involvement.

\section{Conclusion:}

The two cases presented in this case report have similar presentation with fever followed by rapid progressive 
quadriparesis with unconsciousness. This sort of presentation after dog bite and vaccinated status can be explained by paralytic rabies as well as vaccine induced encephalomyelitis. Occasionally it is very difficult to detect clinically the pinpoint diagnosis but the use of NTV is usually followed by encephalomyelitis while the CCV is less likely to do so ${ }^{16}$. The $\mathrm{CCV}$ although expected to have good protection from rabies, the CAT III injury also need HRIG as early as possible to have the best prevention as postexposure prophylaxis. This was proven in our first case as he did not received HRIG but 4 doses of CCV with compliance. The lack of facility to confirm the antigen or even the antibody makes the clinician even more puzzle situation in this sort of case. There need to be good rapport and support from the critical care service for palliative care in paralytic rabies cases as we could not provide appropriately in this case. We still have lack of many neurological investigations especially detecting virus or other organism in CSF and thus make the diagnosis presumptive in many situations including paralytic rabies.

\section{Conflict of Interest : None}

\section{References:}

1. Gamaleia N (1887). Etude sur la rage paralytique chez L'homme. Ann Inst Pasteur (Paris) 1: 63-83.

2. Chopra JS, Banerjee A, Murthy JMK, Pal SR (1980). Paralytic rabies: a clinicopathological study. Brain 103: 789-802

3. Pawan J (1939). Paralysis as a clinical manifestation in human rabies. Ann Trop Med Parasitol33: 21-29.

4. Jackson A (2002). Human disease. In: Rabies. JacksonA, WunnerWH (eds). Amsterdam: Academic Press,pp 219-244.
5. Hemachudha T (1989). Rabies. In: Handbook of clinical neurology viral disease. Vinken P, Bruyn G, Klawans H (eds). Amsterdam: Elsevier Science Publishers, pp 383- 404.

6. Hemachudha T (1994). Human rabies: clinical aspects, pathogenesis, and potential therapy. Curr Top MicrobiolImmunol187: 121-143

7. WHO Expert consultation on Rabies, First report, Technical Report Series 931, 2005;1-121.

8. Amin M R. National Guideline of Rabies Prophylaxis-2010. J Medicine, 2011; 12 : 153-159

9. Warrell DA. The clinical picture of rabies in human. Trans $\mathrm{R}$ Soc Trop Med Hyg. 1976;70(3):188-95.

10. Hemchudha T, Phuapradit P. Rabies. CurrOpinNeurobiol. $1997 ; 10: 260-7$.

11. Rupprecht CE, Hemachudha T (2004). Rabies. In: Infectionsof the central nervous system. Scheld M, Whitley RJ,Marra C (eds). Philadelphia: Lippincott Williams andWilkins, pp 243-259.

12. Laothamatas J, Hemachudha T, Mitrabhakdi E, WannakrairotP, Tulayadaechanont S (2003). MR imagingin human rabies. AJNR Am J Neuroradiol24: 1102-1109.

13. BahriE,LetaiefA, Emez M et al. Neurological complicationsin adults given post exposure Semple type rabies vaccine.Presse Med 1996; 25: 491-3.

14. Briggs DJ, DreesenDW, Wunner WH. Vaccines. In: JacksonAC, WunnerWH, eds. Rabies. San Diego, USA: AcademicPress, 2002; 371-400.

15. C Shayam, AK Duggal, UlkaKamble, AK Agarwal.Postexposure Prophylaxis for Rabies.JIACM 2006; 7(1): 39-46

16. Rupprecht CE, Gibbons RV. Prophylaxis against Rabies. N Engl J Med 2004; 351 (25): 2626-35. 